\title{
The Freeze-All Strategy Revisited: New Pieces to the Old Puzzle
}

\author{
Jan Tesarik* \\ MARGen Clinic, Granada, Spain
}

Submission: March 02, 2021; Published: March 05, 2021

*Corresponding author: Jan Tesarik, MARGen Clinic, Granada, Spain

\begin{abstract}
The development of human embryo freezing techniques was initially motivated by the improvement of ovarian stimulation protocols, leading to the formation of more embryos all of which could not be transferred simultaneously because of the risk of multiple pregnancies. Accordingly, some embryos (usually the best-appearing ones) were transferred in the fresh state, and the remaining ones were frozen to be transferred later. This "fresh-and-freeze" practice was later challenged by two important findings. First, the avoidance of embryo transfer in the ovarian stimulation cycle was shown to reduce the risk of ovarian hyperstimulation syndrome (OHSS). Second, endometrial receptivity tends to be disturbed by the ovarian stimulation. These findings led to the formulation of the freeze-all strategy, whereby all embryos available are frozen for later transfer. However, there are still no generally accepted guidelines to be used when choosing between the fresh-and-freeze and freeze-all strategy. In this paper, the pros and cons of the freeze-all strategy are outlined, and new data to be added to this old puzzle are highlighted..
\end{abstract}

keywords: Embryo freezing; Frozen embryo transfer; Fresh embryo transfer; Freeze-all strategy; Ovarian hyperstimulation syndrome; Endometrial receptivity; Live birth rate

\section{Background and State of the Art}

The first birth after cryopreservation, thawing and uterine transfer of an eight-cell human embryo was reported in 1983 [1]. The interest in applying methods of embryo cryopreservation, previously used successfully in the mouse model [2,3], to human embryos was related to the development of more effective ovarian stimulation protocols, leading to the recovery of more oocytes and embryos that could not be all transferred in the fresh state because of the risk of multiple pregnancies [4]. Accordingly, the use of cryopreserved embryos in human assisted reproduction was originally limited to remaining extra embryos after previous fresh embryo transfer.

The concept of freeze-all strategy, whereby none of the embryos resulting from an in vitro fertilization (IVF) attempt is transferred in the fresh state and all of them are cryopreserved for later transfer, has evolved over the past ten years, mainly for two reasons. First, embryo transfer and pregnancy in an ovarian stimulation cycle can produce ovarian hyperstimulation syndrome (OHSS) in patients at risk, a condition much less likely to occur when embryo transfer is postponed [5]. The second reason is related to the endometrium receptivity for implanting embryos after ovarian stimulation. In fact, ovarian stimulation enables excess number of oocytes to be obtained, but at the same time it impairs endometrium receptivity owing to supraphysiologically high hormone levels during the follicular phase [6]. Moreover, all of the currently used ovarian stimulation protocols also disturb the implantation process after fresh embryo transfer through altering the secretion of progesterone by the corpus luteum, even though this drawback can be partially overcome by an adequate luteal phase support, adapted to the ovarian stimulation protocol used and to the individual characteristics of each patient [7]. In fact, several studies [8-10] reported an improvement of live birth rate (LBR) after frozen embryo transfer (FET) as compared with fresh embryo transfer.

Nevertheless, some other studies comparing fresh and frozen embryo transfers were less conclusive as to the overall beneficial effect on embryo implantation in the whole population of infertile women. A Cochrane systematic review [11] found evidence showing seemingly no difference between the two strategies in cumulative live birth rate per woman. This appears to be due to the fact that some women can benefit from frozen embryo transfer more than others, depending on their response to ovarian stimulation. Analysis of data (2014-1015) from the American Society for Assisted Reproductive Technology Registry showed that frozen embryo transfers benefited only patients who 
produced a large number of oocytes $(>14)$, whereas live birth rates in intermediate and poor responders to ovarian stimulation tended to be higher after fresh embryo transfer [12]. However, these data have to be interpreted with caution because of improvements of embryo freezing and thawing techniques achieved since the time period analyzed so far today.

In addition to OHSS and LBR, however, there are also other issues that need to be taken into account when deciding between the fresh-and-freeze and the freeze-all strategy [13]. Most of these issues concern the obstetric and neonatal outcomes with the use of each of the two strategies. Acccording to a recent metaanalysis [14], pregnancies resulting from FET were associated with lower relative risks of placenta previa, placental abruption, low birth weight, very low birth weight, very preterm birth, small for gestational age, and perinatal mortality compared with fresh embryo transfer. On the other hand, pregnancies occurring from FET were associated with increased risks of pregnancy-induced hypertension, postpartum hemorrhage, and large for gestational age compared with fresh embryo transfer, while the risks of gestational diabetes mellitus, preterm premature rupture of the membranes, and preterm birth showed no differences between the two groups [14]. Apparently, the advantages and disadvantages of FET still remain to be re-evaluated by larger prospective studies. As a precautinary measure they should be ponderated against each other, with regard to the individual condition of each patient.

\section{In search for the most efficient strategy}

In view of the above data, the decision of whether to perform fresh embryo transfer in an ovarian stimulation cycle, with eventual freezing of supernumerary embryos, or whether to freeze all embryos available and postpone embryo transfer for a later date still remains a difficult choice. The decision in favor of the freeze-all option is relatively easy when there is an imminent risk of OHSS or if the patient's endometrium shows apparent irregularities, often accompanied by premature rise of serum progesterone concentration, revealed by ultrasound scan. In most other cases, the decision is not easy and sometimes resembles a puzzle in which some pieces are still lacking. Hence, any new data that can be useful for taking this difficult decision are welcome.

A recent systematic review showed that retrieval of 12 18 oocytes is associated with maximal LBR after fresh embryo transfer, whereas a continuing positive association between the number of oocytes retrieved and cumulative LBR (including the fresh and frozen embryo transfers) was found [15]. The reason of the decrease in LBR after fresh embryo transfer in cases with very high numbers of retrieved oocytes appears to be related with an impairment of uterine receptivity. These data are in agreement with the conclusions of a previously published multicenter randomized controlled trial comparing the efficacy of fresh and frozen single-blastocyst transfers [10].

Assuming that the hyper-response to ovarian stimulation decreases uterine receptivity, it appears reasonable to advise women at risk of this condition about the inconveniences of fresh embryo transfer, also including the risk of OHSS, and to suggest an alternative therapeutical plan. This should be based on adequate ovarian stimulation, aimed at the retrieval of the optimal number (12-18) of oocytes. The subsequent decision as to the embryo transfer strategy (fresh plus frozen or freeze-all) is to be taken with regard to the basic hormonal characteristics of the patient, the course of her ovarian stimulation treatment, and the current aspect of her endometrium. If the freeze-all option is chosen, the subsequent FET should be planned, using a strictly personalized endometrial preparation protocol, similar to that used with success in oocyte donation treatment cycles [16]. This strategy is expected to take full advantage of the strong ovarian response, while limiting the risk of OHSS, preventing the loss of the best embryos due to inadequate uterine receptivity, and thus reducing the need for future ovarian stimulation cycles with their associated cost and discomfort.

\section{References}

1. Trounson A, Mohr L (1983) Human pregnancy following cryopreservation, thawing and transfer of an eight-cell embryo. Nature 305(5936): 707-709.

2. Wilmut I (1972) The effect of cooling rate, warming rate and cryoprotective agent, and stage of development on survival of mouse embryos during freezing and thawing. Life Sci 11(22): 1071-1079.

3. Whittingham DG, Leibo SP, Mazur P (1972) Survival of mouse embryos frozen to $-1960 \mathrm{C}$ and $-2690 \mathrm{oC}$. Science 178(4059): 411-414.

4. Tesarik J (2019) Forty years of in vitro fertilisation: a history of continuous expansion. In: "40 Years After In Vitro Fertilisation: State of the Art and New Challenges. In: J. Tesarik (ed.), Cambridge Scholars Publishing, Newcastle upon Tyne, UK, pp. 1-24.

5. Griesinger G, Schultz L, Bauer T, Broessner A, Frambach T, et al. (2011) Ovarian hyperstimulation syndrome prevention by gonadotropinreleasing hormone agonist triggering of final oocyte maturation in a gonadotropin-releasing hormone antagonist protocol in combination with a "freeze-all" strategy: a prospective multicentric study. Fertil Steril 95(6): 2029-2033.

6. Papanikolaou E, Chartomatsidou T, Timotheou E, Tatsi P, Katsoula E, et al. (2019) In freeze-all strategy, cumulative live birth rate (CLBR) is increasing according to the number of blastocysts formed in women $<40$ undergoing intracytoplasmic sperm injection (ICSI). Front Endocrinol (Lausanne) 10: 427.

7. Tesarik J, Conde-López C, Galán-Lázaro M, Mendoza-Tesarik R (2020) Luteal phase in assisted reproductive technology. Front Reprod Health 2: 595183.

8. Roque M, Lattes K, Serra S, Solà I, Geber S, et al. (2013) Fresh embryo transfer versus frozen embryo transfer in in vitro fertilization cycles: a systematic review and meta-analysis. Fertil Steril 99(1): 156-162.

9. Evans J, Hannan NJ, Edgell TA, Vollenhoven BJ, Lutjen PJ, et al. (2014) Fresh versus frozen embryo transfer: backing clinical decisions with scientific and clinical evidence. Hum Reprod Update 20(6): 808-821.

10. Wei D, Liu J-Y, Sun Y, Shi Y, Zhang B, et al. (2019) Frozen versus fresh single blastocyst transfer in ovulatory women: a multicentre, randomised controlled trial. Lancet 393(10178): 1310-1318.

11. Zaat T, Zagers M, Mol F, Goddijn M, Wely VM, et al. (2021) Fresh versus frozen embryo transfers in assisted reproduction. Cochrane Database of Systematic Reviews 2: CD011184. 
12. Acharya KS, Acharya CR, Bishop K, Harris B, Raburn D, et al. (2018) Freezing of all embryos in in vitro fertilization is beneficial in high responders, but not intermediate and low responders: an analysis of 82,935 cycles from the Society for Assisted Reproductive Technology registry. Fertil Steril 110(5): 880-887.

13. Orvieto R, Kirshenbaum M, Gleicher N (2020) Is Embryo Cryopreservation Causing Macrosomia-and What Else? Front Endocrinol (Lausanne) 11: 19.

14. Sha T, Yin X, Cheng W, Massey IY (2018) Pregnancy-related complications and perinatal outcomes resulting from transfer of cryopreserved versus fresh embryos in vitro fertilization: a metaanalysis. Fertil Steril 109(2): 330-342.

15. Law, YJ, Zhang N, Kolibianakis EM, Costello MF, Keller E, et al. (2021) Is there an optimal number of oocytes retrieved at which live birth rates or cumulative live birth rates per aspiration are maximized after ART? A systematic review. Reprod Biomed Online 42(1): 83-104.

16. Tesarik J (2018) Customised oocyte donation enhancement and new findings regarding the role of growth hormone. Eur Med J 3(4): 87-94.

\section{Your next submission with Juniper Publishers will reach you the below assets}

- Quality Editorial service

- Swift Peer Review

- Reprints availability

- E-prints Service

- Manuscript Podcast for convenient understanding

- Global attainment for your research

- Manuscript accessibility in different formats

( Pdf, E-pub, Full Tsext, Audio)

- Unceasing customer service

Track the below URL for one-step submission https://juniperpublishers.com/online-submission.php 